\title{
Broadband MFP: coherent vs. incoherent
}

\author{
S.M. Jesus and C. Soares \\ SiPLAB-FCT, Universidade do Algarve, Campus de Gambelas, \\ PT-8000 Faro, Portugal.
}

\begin{abstract}
Matched-Field Processing (MFP) is now a mature technique for source localization and tracking. There are at least two aspects that emerge, by their relevance, to the success of MFP: one is the ability of a given MFP processor to accurately pinpoint the source location while rejecting sidelobes, and the other is the impact of erroneous or missing environmental information (known as model mismatch) in the final source location estimate.

This study addresses the first aspect regarding sidelobe rejection while considering that the processor is working on a mismatch free situation. One well known procedure to reduce sidelobes is to use a broadband MFP processor (whenever a band of frequencies is available). There are a number of different ways to combine MFP information across frequency that can be classified in two broad groups: the conventional incoherent methods, that are based on the direct averaging of the auto-frequency inner products and the, say, less conventional methods, that perform a weighted average of the cross-frequency inner products where the weights are the frequency compensation phase-shifts. The later are generally termed as coherent broadband methods since they combine complex inner products.

The coherent broadband methods proposed in the literature are either suboptimal or very computationally intensive, even for a small number of frequencies. An alternative method is presented that combines cross-frequency information with the same localization performance than the standard coherent methods and a computation load similar to that of the incoherent processor. The performance of the various broadband processors is compared in simulated data.
\end{abstract}

Keywords - Matched-field processing, Bartlett processor, broadband source localization, coherent and incoherent estimators.

\section{INTRODUCTION}

The introduction of physical models in underwater acoustics signal processing has been one the most significant advances ever in this field [1],[2]. Defining a physical model for a given practical scenario allows for a consistent inclusion of apriori information on the signal estimation processor. That apriori information consists on the environmental characteristics of the given propagation scenario which, by means of the solution of the wave equation on that scenario, restricts the received acoustic pressure to a well defined class of expected signals. Is that reduction of the class of expected signals that provides the highest gain in terms of parameter estimation performance.

Since the definition of a physical model requires the knowledge (or the assumption) of a number of physical environmentaly measurable quantities, the performance of the processor becomes dependent on those quantities. Conversely, if the emitted and received signals are known (or

This work was supported under projects ATOMS, contract PDCTM/P/MAR/15296/1999, FCT, Portugal and TOMPACO, CNR, Italy. measurable) than it is, in principle, possible to estimate the environmental characteristics of the media of propagation - that is the base of the various matched-field $(M F)$ based techniques being developed in the last two decades: Matched-Field Processing for source localization, Matched-Field Tomography for ocean acoustic tomography and Matched-Field Inversion for geoacoustic parameter estimation.

There are at least two aspects that emerge by their relevance to the success of MF based techniques: one is the ability of a given MF processor to accurately pinpoint the source location while rejecting sidelobes, and the other is the impact of erroneous or missing environmental information (known as model mismatch) in the final parameter estimate. This study addresses the first aspect regarding sidelobe rejection while considering that the processor is working on a mismatch free situation. In that case, the capacity of separating "closely" spaced acoustic fields (the so-called discrimination) largely depends on the degree of complexity of the received acoustic pressure field. As an example, a single tone will have two parameters for matching: the amplitude and the phase. If a broadband signal is transmitted, there are as many amplitudes and phases as frequencies, and the complexity of the received signal is increased naturally leading to a higher MF discrimination.

There are a number of different ways to combine MF information across frequency that can be classified in two broad groups: the conventional incoherent methods [3] that are based on the direct averaging of the auto-frequency inner products (average of real numbers) and the, say, less conventional methods [4]-[10], that perform a weighted average of the cross-frequency inner products where the weights are the frequency compensated phase-shifts. The later are generally called coherent broadband methods since they combine complex inner products.

Despite the considerable amount of work on broadband methods there is a lack of understanding on why and when a coherent method provides a better detection or localization performance than an incoherent method. This is the main topic addressed in the present study, that starts by presenting a physical-based linear data model with suitable random perturbation terms. Under this model it is shown that the advantage of using the cross-frequency terms resides in its ability to reject noise while its disadvantage is that the result is limited by the correlation of the random phase terms across frequency. An efficient algorithm for combining cross-frequency information is derived that is shown to have an equivalent localization performance than the optimal coherent processor with a computation burden similar to that of the incoherent processor. Then, the 
performance of the coherent and incoherent processors are compared for different number of frequencies using simulated data.

\section{THEORETICAL BACKGROUND}

\section{A. The physical-based data model}

The normally assumed data model for $M$ farfield point sources emitting narrowband signals received in a L-sensor receiving array is given by

$$
\mathbf{x}(t)=\mathbf{A}(\theta) \mathbf{s}(t)+\mathbf{u}(t)
$$

where $\mathbf{x}(t)$ is the L-sensor array received acoustic pressure, $\mathbf{A}(\theta)$ is the $L \times M$ steering matrix, which entries are the appropriate delays for each array sensor and each source $m$ at (unknown) source bearing $\theta_{m}, \mathbf{s}(t)$ is the $M$-dimensional vector with the sources input at time $t$ and $\mathbf{u}(t)$ is the observation additive noise. A common assumption is to consider that the noise is white, Gaussian, zero mean and uncorrelated with the signals $\mathbf{s}(t)$, that themselves are zero mean and uncorrelated stochastic processes. This model is usefull for describing a field of independent noise sources propagating through a non dispersive unbounded media and received on an horizontal array. When dealing with shallow water dispersive scenarios, deterministic sources and non horizontal arrays this model is uncapable to account for the complexity of the received field as a mixture of correlated (partially) deterministic signal reflections from sea bottom and sea surface.

Introducing a realistic physical model description requires considerable modification of data model (1). Under a physical-based approach, there is a deterministic signal term that contains all the unknown true parameters $\theta_{o}$ and an additive, zero-mean, white noise term, such that the Lsensor received acoustic pressure due to an harmonic source at frequency $\omega$ is

$$
\mathbf{x}\left(\theta_{o}\right)=\mathbf{h}\left(\theta_{o}\right) s+\mathbf{u}
$$

where $\mathbf{h}\left(\theta_{o}\right)$ is an $L$-dimensional vector containing the channel impulse response structure for the $L$ receivers, $s$ is the source signal spectrum at frequency $\omega$ and $\mathbf{u}$ is the L-dimensional observation noise vector, assumed spatially white with variance $\sigma_{u}^{2}$. In the case of $M>1$ sources, the signal term would become $\mathbf{H}\left(\theta_{o}\right) \mathbf{s}$, where $\mathbf{H}\left(\theta_{o}\right)$ is now an $L \times M$ matrix and $\mathbf{s}$ is an $M$-dimensional vector. This paper addresses the case of a single source, while the multiple source case can be easily deduced by the appropriate changes.

In order to obtain a meaningfull observation of the received acoustic pressure it is acceptable to introduce some variability on the physical signal model. Assuming that the randomness of the propagation media affects equally all receivers (a secondary effect of the farfield assumption) it is acceptable to introduce a scalar complex random variable $p=|p| \exp (j \phi)$, (see for example [3]) as

$$
\mathbf{x}\left(\theta_{o}\right)=p \mathbf{h}\left(\theta_{o}\right) s+\mathbf{u},
$$

where the noise $\mathbf{u}$ is assumed to be uncorrelated with the signal perturbation $p$. There is some trepidation on the assumed distribution for the random factor $p$ on (3).

The correlation matrix can be directly written from (3) as

$$
\begin{aligned}
\mathbf{C}_{x x} & =E\left[\mathbf{x x ^ { H }}\right] \\
& =E\left[|p|^{2}\right]|s|^{2} \mathbf{h}\left(\theta_{o}\right) \mathbf{h}^{H}\left(\theta_{o}\right)+\sigma_{u}^{2} \mathbf{I},
\end{aligned}
$$

where all terms have been previously defined and ${ }^{H}$ denotes conjugate transpose. Equation (3) gives the essential description of the received field data model in the narrowband case. When a time-limited signal (impulse) is transmitted from the source, a significant band of frequencies of the acoustic channel are excited giving rise to the need for a broadband formulation. In order to introduce, as much as possible, a common frame for the narrowband and broadband cases we define an extended vector such as

$$
\underline{\mathbf{x}}=\left[\mathbf{x}^{T}\left(\omega_{1}\right), \mathbf{x}^{T}\left(\omega_{2}\right), \ldots, \mathbf{x}^{T}\left(\omega_{K}\right)\right]^{T},
$$

where ${ }^{T}$ denotes matrix transpose and $K$ is the total number of discrete frequency bins. In that case, the broadband model can be written as

$$
\underline{\mathbf{x}}=\mathbf{H}\left(\theta_{o}\right) \underline{\tilde{\mathbf{s}}}+\underline{\mathbf{u}}
$$

where $\underline{\tilde{\mathbf{s}}}$ is a $\mathrm{K}$-dimensional random vector which entries are $s\left(\omega_{k}\right) p\left(\omega_{k}\right)$, i.e., the source spectrum multiplied by the random perturbation factor at each frequency $\omega_{k} \in\left[\omega_{1}, \omega_{K}\right]$; the matrix $\underline{\mathbf{H}}\left(\theta_{o}\right)$ is

$$
\mathbf{H}\left(\theta_{o}\right)=\left[\begin{array}{cccc}
\mathbf{h}\left(\omega_{1}, \theta_{o}\right) & 0 & \ldots & 0 \\
0 & \mathbf{h}\left(\omega_{2}, \theta_{o}\right) & \ldots & 0 \\
\vdots & \vdots & \ddots & \vdots \\
0 & 0 & \ldots & \mathbf{h}\left(\omega_{K}, \theta_{o}\right)
\end{array}\right],
$$

where the explicit frequency dependency of $\mathbf{h}\left(\omega, \theta_{0}\right)$ has been included and the noise extended vector $\mathbf{u}$ has an obvious notation similar to (6). It is interesting to write the correlation matrix for model (7), which cross-frequency block matrix is given by

$$
\begin{aligned}
& \mathbf{C}_{x x}\left(\omega_{i}, \omega_{j}\right)= \\
& \begin{cases}s\left(\omega_{i}\right) s^{*}\left(\omega_{j}\right) \mathbf{h}\left(\omega_{i}, \theta_{o}\right) \mathbf{h}^{H}\left(\omega_{j}, \theta_{o}\right) E\left[p\left(\omega_{i}\right) p^{*}\left(\omega_{j}\right)\right] & i \neq j \\
\left|s\left(\omega_{i}\right)\right|^{2} \mathbf{h}\left(\omega_{i}, \theta_{o}\right) \mathbf{h}\left(\omega_{i}, \theta_{o}\right)^{H} E\left[\left|p\left(\omega_{i}\right)\right|^{2}\right]+\sigma_{u}^{2} \mathbf{I} & i=j,\end{cases}
\end{aligned}
$$

where the term $E\left[p\left(\omega_{i}\right) p^{*}\left(\omega_{j}\right)\right]$ denotes the correlation of the perturbation factor across frequency. Note that unlike the auto-frequency entries $(i=j)$ the cross-frequency terms $(i \neq j)$ are noise free. This is due to the well known property of the Fourier transform for time stationnary processes that gives uncorrelated cross-frequency bins. In practice, with finite time observation that property is only assymptotically verified, which is sufficient for practical purpose. 


\section{B. The Bartlett processor}

The Bartlett processor is possibly the most widely used estimator in MF parameter identification. The parameter estimate $\hat{\theta}_{o}$ is given as the argument of the maximum of the functional

$$
P(\theta)=E\left[\hat{\mathbf{w}}^{H}(\theta) \mathbf{x}\left(\theta_{o}\right) \mathbf{x}^{H}\left(\theta_{o}\right) \hat{\mathbf{w}}(\theta)\right],
$$

where the replica vector estimator is determined as the vector $\mathbf{w}(\theta)$ that maximizes the mean quadratic power,

$$
\hat{\mathbf{w}}(\theta)=\arg \max _{\mathbf{w}} E\left[\mathbf{w}^{H}(\theta) \mathbf{x}\left(\theta_{o}\right) \mathbf{x}^{H}\left(\theta_{o}\right) \mathbf{w}(\theta)\right],
$$

subject to $\mathbf{w}^{H}(\theta) \mathbf{w}(\theta)=1$. In the narrowband case, using model (3) gives the well known non-trivial solution

$$
\hat{\mathbf{w}}_{N B}(\theta)=\frac{\mathbf{h}(\theta)}{\sqrt{\mathbf{h}^{H}(\theta) \mathbf{h}(\theta)}},
$$

where the denominator is a normalization scalar and the numerator contains the signal structure as "seen" at the receiving array. This is simply the classical matched-filter for the particular parameter location $\theta$. If the search is made over $\theta$ and the maximum is selected, then an optimum mean least-squares estimate $\hat{\theta}_{o}$ of $\theta_{o}$ is obtained.

In the broadband case, the estimator of the replica vector is given in terms of frequency extended vectors using model (7), thus

$$
\underline{\hat{\mathbf{w}}}_{B B}=\arg \max _{\underline{\mathbf{w}}}\left\{\underline{\mathbf{w}}^{H} \mathbf{H}\left(\theta_{O}\right) E\left[\underline{\tilde{\mathbf{s}}}^{H}\right] \mathbf{H}^{H}\left(\theta_{o}\right) \underline{\mathbf{w}}\right\},
$$

where the expectation of the signal matrix relates to the correlation of the perturbation factor $p$ across frequency, weighted by the source power cross-spectrum $s\left(\omega_{i}\right) s\left(\omega_{j}\right)$. No closed form for the replica vector can be given in this case without explicit knowledge of that matrix. There are a number of possible implementations that represent suboptimal versions of (13) with different assumptions for the structure of the signal matrix.

\section{Broadband incoherent processor}

The so-called incoherent broadband Bartlett processor assumes that the random factor is simply $E\left[p\left(\omega_{i}\right) p^{*}\left(\omega_{j}\right)\right]=$ $\sigma_{p}^{2} \delta_{i j}$, i.e., uncorrelated across frequency with a flat spectrum, which gives

$$
\underline{\hat{\mathbf{w}}}_{\mathrm{inc}}(\theta)=\frac{\mathbf{H}(\theta) \underline{\tilde{\mathbf{s}}}}{|\mathbf{H}(\theta) \underline{\tilde{\mathbf{s}}}|}
$$

thus, by replacement into (10), gives the processor expression

$$
P_{\text {inc }}(\theta)=\frac{\sum_{k=1}^{K}\left|s\left(\omega_{k}\right)\right|^{2} \mathbf{h}^{H}\left(\omega_{k}, \theta\right) \mathbf{C}_{x x}\left(\omega_{k}, \omega_{k}\right) \mathbf{h}\left(\omega_{k}, \theta\right)}{\sum_{k=1}^{K}\left|\mathbf{h}\left(\omega_{k}, \theta\right) s\left(\omega_{k}\right)\right|^{2}}
$$

which is nothing more than a source power weighted sum of the diagonal matched-filtered auto-frequency block matrices of the extended correlation matrix $\underline{\mathbf{C}}_{x x}$. When the source power weights are unknown, the currently unweighted incoherent processor is a suboptimal estimator for non flat source power spectrum.

\section{Broadband coherent processor}

Although there is good evidence that for many of the underwater propagation channels most of the energy is concentrated along the main diagonal of the cross-spectrum correlation matrix (the auto-frequency terms) it is also clear that the same auto-frequency terms would carry most of the noise power as it can be seen in expression (9). One of the motivations when performing coherent processing is to take advantage of the noiseless cross-frequency terms of (9). This explains why in most studies concernned with coherent processing only the cross-frequency terms were used, excluding the diagonal auto-frequency information. There actually several broadband coherent processors depending on the assumptions made for approximating the cross-frequency perturbation terms of the signal matrix $E\left[\underline{\tilde{\mathbf{s}}}^{H}\right]$ of $(13)$.

\section{D.1 Coherent normalized processor}

The coherent normalized processor has been proposed by michalopoulou [6], [7] and attempts to eliminate the source spectrum-perturbation weighting across frequency. At each frequency $\omega_{i}$ a normalized model vector is defined as

$$
\mathbf{n}_{x}\left(\omega_{i}, \theta_{o}\right)=\frac{\mathbf{x}\left(\omega_{i}, \theta_{o}\right)}{x_{l}\left(\omega_{i}, \theta\right)}
$$

where $x_{l}\left(\omega_{i}, \theta\right)$ is the signal received at sensor $l$. The choice of $l$ depends on the actual signal-to-noise ratio (SNR) at that particular sensor. In a high SNR situation, if the noise contribution at sensor $l$ is neglected, the normalized data model becomes

$$
\mathbf{n}_{x}\left(\omega_{i}, \theta\right) \approx \mathbf{n}_{h}\left(\omega_{i}, \theta_{o}\right)+\frac{\mathbf{u}\left(\omega_{i}\right)}{h_{l}\left(\omega_{i}, \theta_{o}\right) s\left(\omega_{i}\right) p\left(\omega_{i}\right)}
$$

Matching this model with an extended normalized replica vector yields a perfect match for the signal structure while a strongly correlated structure for the noise field due to the second term of (17). In that case the coherent normalized replica vector is written as

$$
\hat{\mathbf{w}}_{\mathrm{coh}-\mathrm{n}}\left(\omega_{i}, \theta\right)=\mathbf{n}_{h}\left(\omega_{i}, \theta\right)=\frac{\mathbf{h}\left(\omega_{i}, \theta\right)}{h_{l}\left(\omega_{i}, \theta\right)}
$$

and using that expression in the Bartlett processor gives

$$
\begin{aligned}
P_{\mathrm{coh}-\mathrm{n}}(\theta)= & \sum_{i=1}^{K} \sum_{j=1}^{K} \mathbf{n}_{h}^{H}\left(\omega_{i}, \theta\right) \mathbf{n}_{h}\left(\omega_{i}, \theta_{o}\right) \mathbf{n}_{h}^{H}\left(\omega_{j}, \theta_{o}\right) \mathbf{n}_{h}\left(\omega_{j}, \theta\right) \\
& +\mathbf{n}_{h}^{H}\left(\omega_{i}, \theta\right) \mathbf{C}_{n_{u} n_{u}}\left(\omega_{i}, \omega_{j}\right) \mathbf{n}_{h}\left(\omega_{j}, \theta\right)
\end{aligned}
$$

which shows a perfectly coherent match for the signal model part when $\theta=\theta_{o}$, and a noise term residual which is a constant when $i=j$, due to the white noise assumption, and has a correlation structure for $i \neq j$ that is highly dependent on the cross-frequency correlation of the perturbation $p(\omega)$. 
(a)

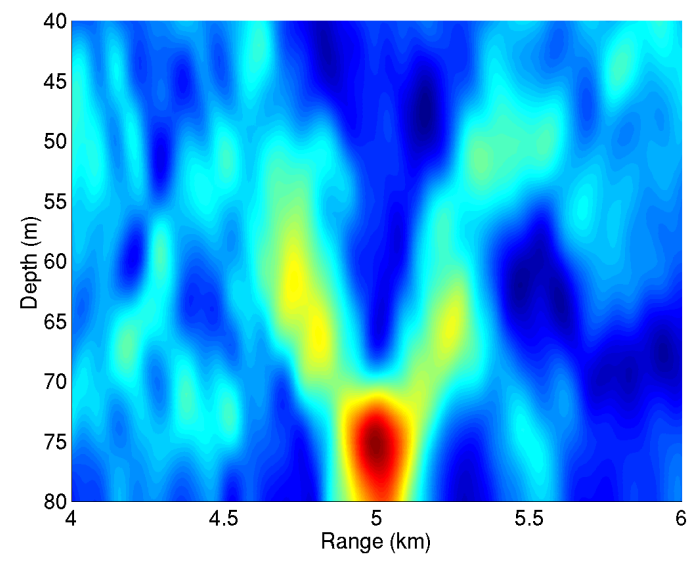

(c)

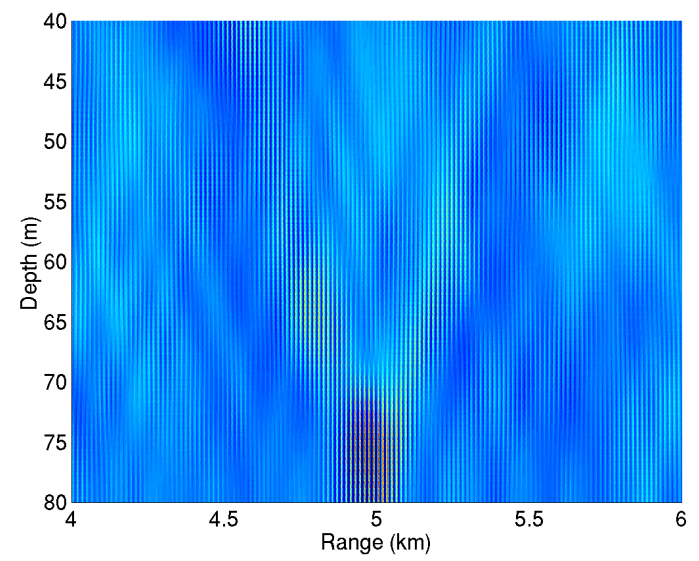

(b)

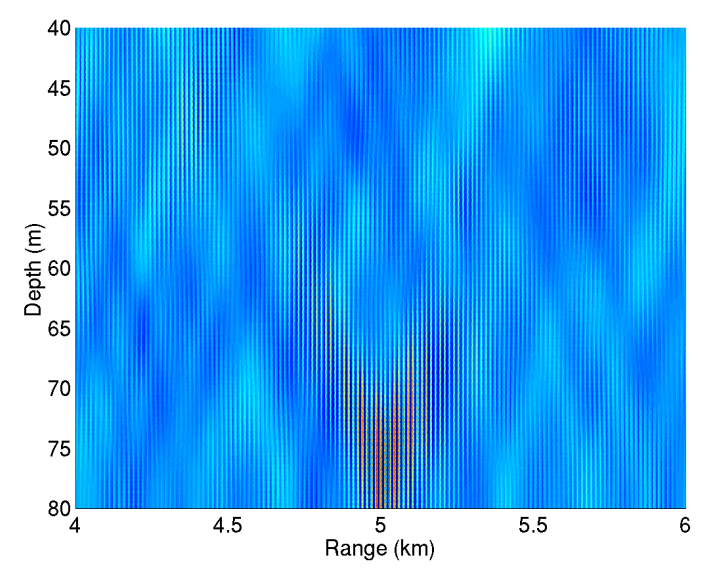

(d)

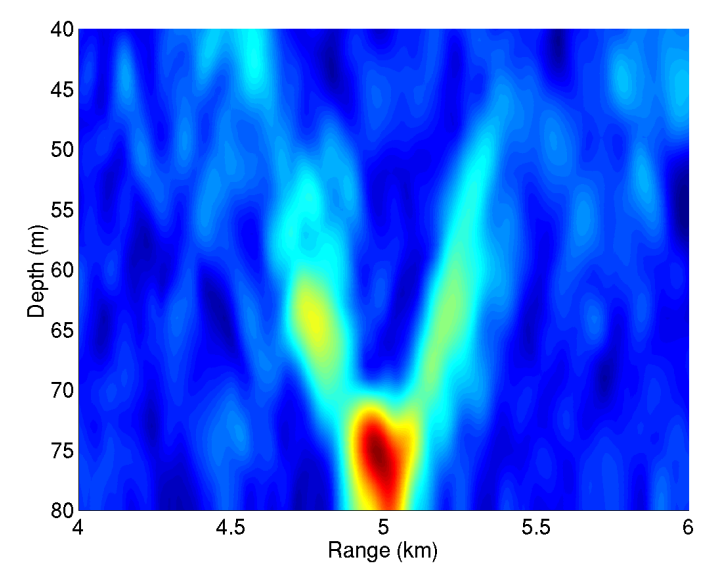

Fig. 1. Range-depth ambiguity surfaces computed with synthetic data for frequencies $300,400,500$, and $600 \mathrm{~Hz}, \mathrm{SNR}=-8 \mathrm{~dB}$ and the following processors: incoherent (a), normalized coherent(b), matched-phase coherent(c), and incoherent cross-frequency(d).

\section{D.2 Matched-phase coherent processor}

Another approximation to the broadband coherent processor has been recently proposed by Orris [9] where the correlation terms are explicitely included in the replica vector as unknowns and have therefore to be estimated. A new replica vector is therefore defined as

$$
\underline{\mathbf{w}}_{\mathrm{coh}-\mathrm{mp}}(\theta)=\left[\mathbf{h}^{T}\left(\omega_{1}, \theta\right) e^{\hat{\phi}_{h}\left(\omega_{1}\right)}, \ldots, \mathbf{h}^{T}\left(\omega_{K}, \theta\right) e^{\hat{\phi}_{h}\left(\omega_{K}\right)}\right]^{T}
$$

where the phase terms $\left[\hat{\phi}_{h}\left(\omega_{k}\right) ; k=1, \ldots, K\right]$ are the estimates that maximize the output power upon summation over sensor and frequency. Taking into account that, when carrying out that summation, each term has its complex conjugate, the energy contained in the imaginary part is lost. The unknown phase terms $\phi_{h}$ are estimated in such a way as to minimize that loss which, ideally, requires the unknown phase terms to be symmetric to the phase of the signal matrix terms on (9). If that is achieved all terms turn into real numbers and the sum is carried out in phase. In that case, and for a flat spectrum source, this processor is optimum. Replacing (20) in the Bartlett processor expression gives

$$
\begin{array}{r}
P_{\text {coh }-\mathrm{mp}}(\theta)=\sum_{i=1}^{K} \sum_{l=1}^{K} \mathbf{h}^{H}\left(\omega_{i}, \theta\right) \mathbf{C}_{x x}\left(\omega_{i}, \omega_{l}\right) \mathbf{h}\left(\omega_{l}, \theta\right) \\
e^{-j\left[\hat{\phi}_{h}\left(\omega_{i}\right)-\hat{\phi}_{h}\left(\omega_{l}\right)\right]}
\end{array}
$$

In practice, the problem mentioned by Orris [9], is the computation load for an exhaustive search over the search space of the order of $o=J^{K} \times M \times N$, where $J$ is the number of samples for the phase in $[0,2 \pi], K$ is the number of frequencies and $M \times N$ is the $\theta$ parameter search grid (e.g. range vs. depth). In practice, and as mentioned by Orris [9], computation complexity limits the number of frequencies to $K=3$.

\section{E. The cross-frequency incoherent processor}

The cross-frequency incoherent processor is an alternative to overcome the computational burden of the matchedphase processor while keeping the same performance. This processor steams from the simple idea that the phase corrections for the surface maximum $\left(\theta=\theta_{o}\right)$ are

$$
\phi_{h}\left(\omega_{i}\right)-\phi_{h}\left(\omega_{j}\right)=\angle s\left(\omega_{i}\right) s^{*}\left(\omega_{j}\right) E\left[p\left(\omega_{i}\right) p^{*}\left(\omega_{j}\right)\right],
$$


for all $i, j=1, \ldots, K$ which can be seen by direct inspection of (9) and where $\angle$ means "phase of". When these corrections are set the value of the maximum is just the sum of a series of real numbers, which are the squared modules of the quadratic forms across frequency, i.e.,

$$
P_{\text {inc }-\mathrm{xf}}(\theta)=\sum_{i=1}^{K} \sum_{j=1}^{K}\left|\mathbf{h}^{H}\left(\omega_{i}, \theta\right) \mathbf{C}_{x x}\left(\omega_{i}, \omega_{j}\right) \mathbf{h}\left(\omega_{j}, \theta\right)\right|^{2}
$$

The value of the peak for the true source location obtained with (23) is exactly the same as that obtained with (21) with absolutely no parameter search ! Therefore, the peak would have the same height and the same location, however the aspect of the resulting surface would be much different from the cross-frequency and the matched-phase processors: the former would have a smooth appearance much like the incoherent processor and the later would have extremely narrow peaks distributed along the surface with an overall envelope that is very similar to that of the crossfrequency incoherent processor. That will be exemplified in the simulation examples.

\section{Simulation RESUlts}

This section shows a few simulated data examples of the application of the MF processors shown above to source localization. The data was simulated using the C-SNAP model [11] in a $80 \mathrm{~m}$ deep range-independent shallow water scenario. The acoustic source is placed at $76 \mathrm{~m}$ depth and $5 \mathrm{~km}$ range from a 32 -sensors vertical array. The source is emitting a series of multitones between 300 and $600 \mathrm{~Hz}$. The correlation matrix was estimated using 32 snapshots that were generated with an SNR of $0 \mathrm{~dB}$. Figure 1 shows the range-depth ambiguity surfaces obtained for the above referred broadband Bartlett processors, $P_{\text {inc }}$ (a), $P_{\text {coh }-\mathrm{n}}(\mathrm{b}), P_{\text {coh }-\mathrm{mp}}(\mathrm{c})$ and $P_{\text {inc-xf }}(\mathrm{d})$. In cases (b), (c) and (d) only the cross-frequency terms were used. As expected, the incoherent processors (a) and (d), gave similarly smooth surfaces with a lower sidelobe structure for the cross-frequency processor. The coherent processors (b) and (c) also gave similar responses with a large number of very narrow peaks (up to only $1 \mathrm{~m}$ wide in range!) that are due to a perfect alignement of the surfaces for all grid points. That peaky structure is modulated by the crossfrequency incoherent response over the whole search surface. At low SNR the coherent normalized processor (b), rapidly degradatesdue to the SNR limitation pointed out in (17). As explained above the matched-phase and the cross-frequency coherent processors have analytically the same source detection performance. That performance is shown in figure 2 for the no perturbation model, where the improvement obtained by adding several frequencies can be clearly estimated to be of approximately $5 \mathrm{~dB}$ when passing from 4 to 16 frequencies at usefull detection probabilities. At low and high detection rates the two processors tend to give the same result. In presence of perturbation, the results mainly depend on the cross-frequency perturbation correlation. (a)

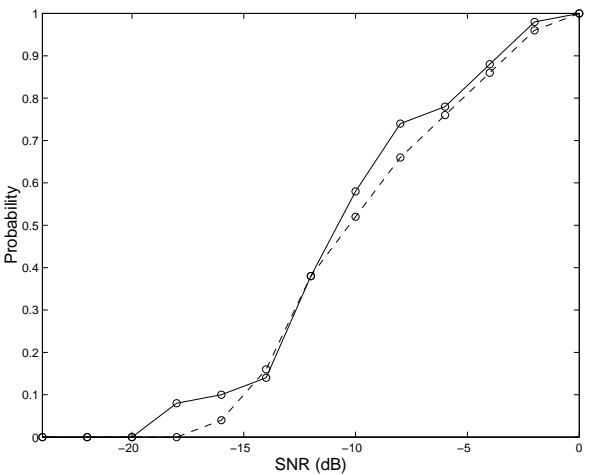

(b)

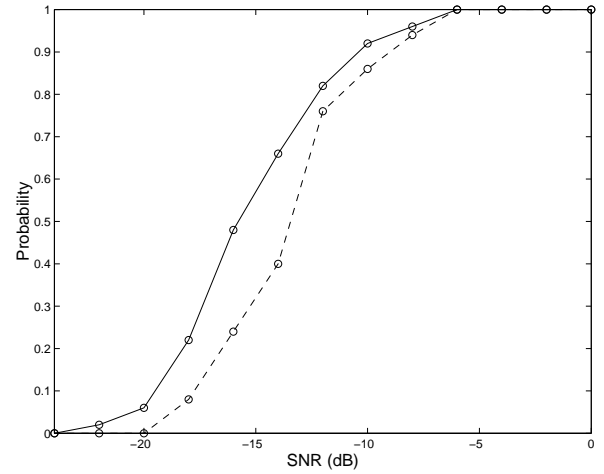

(c)

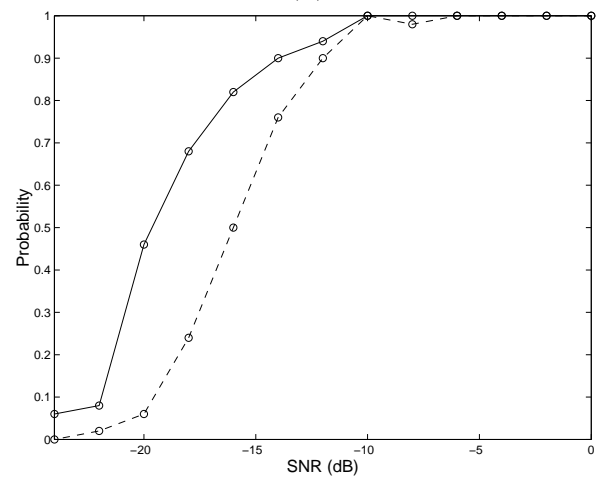

Fig. 2. Estimated probability of correct source localization obtained on a sample of 32 statistical draws, for the incoherent processor (dashed) and the cross-frequency incoherent processor (continuous) for the no perturbation case $p(\omega)=1$ with 4 frequencies (a), 7 frequencies (b) and 16 frequencies (c).

\section{Conclusion}

Physical-based MF techniques have been widely accepted in the underwater acoustics community as usefull tools for estimating the location of a sound source or the geophysical parameters of the propagation media. The first results and developments were performed for narrowband signals that quickly evolved to computer intensive broadband calculations. As in their planewave-array processing counterparts, MF techniques require extensive modification when passing from narrowband to broadband.

Amongst the panoply of methods and processors found in the literature, the Bartlett power estimator has been one of the most widely used. This paper attempts to develop 
a common frame for evaluating three broadband variants of the Bartlett processor and presenting a fourth processor that is shown to have the same performance as the optimum coherent processor with a small fraction of the computational cost.

This work starts by presenting some rationale for the inclusion of a random phase term in the signal part of the linear data model. Under this model it is shown that the advantage of using the cross-frequency terms resides in its ability to reject noise while its disadvantage is that the result is limited by the correlation of the random phase terms across frequency. The well known Bartlett incoherent processor avoids the need for the estimation of that correlation term by using only the auto-frequency terms of the data correlation matrix. In order to take advantage of the cross-frequency terms a frequency normalization algorithm and a direct matched-phase estimation algorithm have been proposed in the literature. The former is limited by the available SNR and the later by an exceptionally high computational complexity. A carefull analysis of the expressions lead to an efficient cross-frequency incoherent algorithm that is able to handle virtually any number of frequencies in a reasonable computation time and amazingly has the same detection performance than the matched-phase coherent processor. Although not shown in this study, the comparative performance of the tested algorithms still holds in presence of model mismatch.

\section{REFERENCES}

[1] H.P. Bucker, "Use of calculated sound fields and matcheddetection to locate a sound source in shallow water", J. Acoust. Soc. America, 59, pp. 368-373, 1976

[2] A.B. Baggeroer, W.A. Kuperman and P.N. Mikhalewsky, "An overview of matched-field methods in ocean acosutics", IEEE J. Oceanic Eng., 18, pp. 401-424, 1993.

[3] A.B. Baggeroer, W.A. Kuperman and H. Schmidt, "Matchedfield processing: source localization in correlated noise ans an optimum parameter estimation problem", J. Acoust. Soc. America, 80, pp. 571-587, 1988.

[4] A. Tolstoy, "Computational aspects of matched field processing in underwater acoustics", in D. Lee, A. Cakmak and R. Vichnevetsky Editors, Computational Acoustics, 3, pp. 303-310, North-Holland, Amsterdam, 1990.

[5] A. Tolstoy, Matched-Field Processing for Underwater Acoustics, World Scientific, Singapore, 1993.

[6] Z.-H. Michalopoulou, "Matched-field processing for broadband source localization", IEEE J. Ocean Eng., 21, pp.384-392, 1996.

[7] Z.-H. Michalopoulou, "Source tracking in the Hudosn canyon experiment", J. Comp. Acoustics, 4, pp.371-383, 1996.

[8] S.P. Cznszak and J.L. Krolik, "Robust wideband matched-field processing with a short vertical array", J. Acoust. Soc. America, 101, pp.749-759, 1997.

[9] G.J. Orris, M. Nicholas and J.S. Perkins, "The matched-phase coherent multi-frequency matched field processor", J. Acoust. Soc. America, 101, pp.749-759, 2000.

[10] Z.-H. Michalopoulou, "Robust multi-tonal matched-field inversion: a coherent approach", J. Acoust. Soc. America, 104, pp. 163-170, 1998.

[11] C.M. Ferla, M.B. Porter e F.B. Jensen, "C-SNAP: Coupled SACLANTCEN nnormal mode propagation loss model", SACLANTCEN SM-274, Saclant Undersea Research Centre, La Spezia, Italy, 1993. 